\title{
A broadbanded pressure differential wave energy converter based on dielectric elastomer generators
}

\author{
Michele Righi - Giacomo Moretti • \\ David Forehand • Lorenzo Agostini • \\ Rocco Vertechy • Marco Fontana [
}

Received: 9 October 2020 / Accepted: 4 May 2021 / Published online: 11 August 2021

(C) The Author(s) 2021

\begin{abstract}
Dielectric elastomer generators (DEGs) are a promising option for the implementation of affordable and reliable sea wave energy converters (WECs), as they show considerable promise in replacing expensive and inefficient power take-off systems with cheap direct-drive generators. This paper introduces a concept of a pressure differential wave energy converter, equipped with a DEG power take-off operating in direct contact with sea water. The device consists of a closed submerged air chamber, with a fluid-directing duct and a deformable DEG power take-off mounted on its top surface. The DEG is cyclically deformed by waveinduced pressure, thus acting both as the power take-off and as a deformable interface with the waves. This layout allows the partial balancing of the stiffness due to the DEG's elasticity with the negative hydrostatic stiffness contribution associated with the displacement of
\end{abstract}

Supplementary Information The online version contains supplementary material available at https://doi.org/10.1007/ s11071-021-06721-8.

M. Righi · G. Moretti · M. Fontana $(\varangle)$

TeCIP Institute, Scuola Superiore Sant'Anna, Pisa, Italy

e-mail: marco.fontana@santannapisa.it

M. Righi

e-mail: righi.michele23@gmail.com

D. Forehand

Institute for Energy Systems, University of Edinburgh,

Edinburgh, UK

L. Agostini · R. Vertechy

Department of Industrial Engineering, University of Bologna, Bologna, Italy the water column on top of the DEG. This feature makes it possible to design devices in which the DEG exhibits large deformations over a wide range of excitation frequencies, potentially achieving large power capture in a wide range of sea states. We propose a modelling approach for the system that relies on potential-flow theory and electroelasticity theory. This model makes it possible to predict the system dynamic response in different operational conditions and it is computationally efficient to perform iterative and repeated simulations, which are required at the design stage of a new WEC. We performed tests on a small-scale prototype in a wave tank with the aim of investigating the fluid-structure interaction between the DEG membrane and the waves in dynamical conditions and validating the numerical model. The experimental results proved that the device exhibits large deformations of the DEG power take-off over a broad range of monochromatic and panchromatic sea states. The proposed model demonstrates good agreement with the experimental data, hence proving its suitability and effectiveness as a design and prediction tool.

Keywords Dielectric elastomers - Wave energy · Fluid-structure interaction · Stiffness compensation

\section{Introduction}

Dielectric elastomers are multifunctional polymeric materials that are employed to develop lightweight and 
low-cost electromechanical transducers, such as actuators or generators [1].

Recently, interest towards dielectric elastomer generators (DEGs) has grown thanks to their potential application as power take-off systems in wave energy converters (WECs). Compared to traditional power takeoff technologies, dielectric elastomers may allow the development of advanced WEC architectures capable of converting wave energy into direct current electricity within a wide range of operating sea conditions. Dielectric elastomers possess a number of attributes that make them a promising technology for the wave energy sector, e.g. high energy density, low cost, high robustness and reliability, intrinsic cyclical operation, lightweight, and silent operation [2].

Several architectures of dielectric elastomer-based WECs have been studied by companies and research groups [2-5]. One of the most widely investigated layouts is the DEG-based oscillating water column [6,7], which holds a set of inflatable DEGs, mounted on top of a semi-submerged collector, as the only moving part. Experimental proof of concept of DEG oscillating water columns has been recently presented, with test campaigns run both in wave tank facilities [7] and in a real sea basin [8]. These tests proved that DEGbased oscillating water columns are potentially capable of efficiently converting wave power, achieving fullscale equivalent power outputs of several hundreds of kilowatts.

In order to capture a significant portion of the incoming wave power, DEG oscillating water columns are required to be dynamically tuned with the incoming waves, i.e. their natural frequency should be close to the wave frequency [9]. To implement resonant designs, the large elastic stiffness introduced by the DEG should be counterbalanced by a sufficiently large hydrodynamic inertia, whose implementation involves complex structural designs [2]. In addition to that, outside of the resonant range, the device's performance significantly drops away.

Here we investigate a design layout which overcomes the limitations of DEG oscillating water columns by taking advantage of direct contact between the DEG and the hydraulic domain. The presented WEC layout is a pressure-differential WEC (PD-WEC) that consists of a submerged air chamber with a horizontally mounted circular DEG at its top, which directly interacts with the wave pressures. Thanks to its particular geometrical layout, this PD-WEC implements a nega- tive hydrostatic stiffness that balances the DEG elastic stiffness, enabling the achievement of large deformations (and, hence, efficient power capture) over a wide range of wave frequencies.

The DEG-based PD-WEC has been conceptually introduced in [2], and it is systematically investigated and tested here for the first time. In this paper, we present a lumped-parameter numerical model of the system, which describes the complex fluid-structure interactions involved in its operation by resorting to a one degree of freedom formulation. This model provides a computationally inexpensive tool to perform device design and assess the convertible power. We then present the results of an experimental campaign in a wave tank facility, aimed at investigating the hydroelastic response of a PD-WEC with a styrenic rubber dielectric elastomer generator [10]. Besides providing validation of the proposed numerical model, the experimental results confirm that the device is able to provide large DEG deformations in response to broadbanded incident waves, thus proving its potential for application in highly variable wave climates.

Since the ability of DEGs to generate electrical energy in dynamic conditions has been proved in the past (for example, by fully functional prototypes $[2,11]$ ), this work is mainly focused on the analysis of the fluidstructure-interaction aspect of the device under investigation, the response of which is characterised by highly coupled and nonlinear dynamics.

The paper is structured as follows. Section 2 describes the PD-WEC layout and illustrates the associated negative hydrostatic stiffness mechanism. Section 3 presents a lumped-parameter model which describes the nonlinear hydroelectroelastic interactions in a PDWEC via a single degree of freedom parametrisation. Section 4 describes the design of a prototype and the implementation of wave tank experiments aimed at the investigation of the fluid-structure interaction. Section 5 presents the experimental wave tank results and provides validation of the proposed model. Section 6 presents the conclusions.

\section{Device layout and working principle}

The PD-WEC has an axisymmetric shape and holds a circular diaphragm dielectric elastomer membrane (or stack of membranes), coated by compliant electrodes, radially pre-stretched and clamped on the top of a sub- 


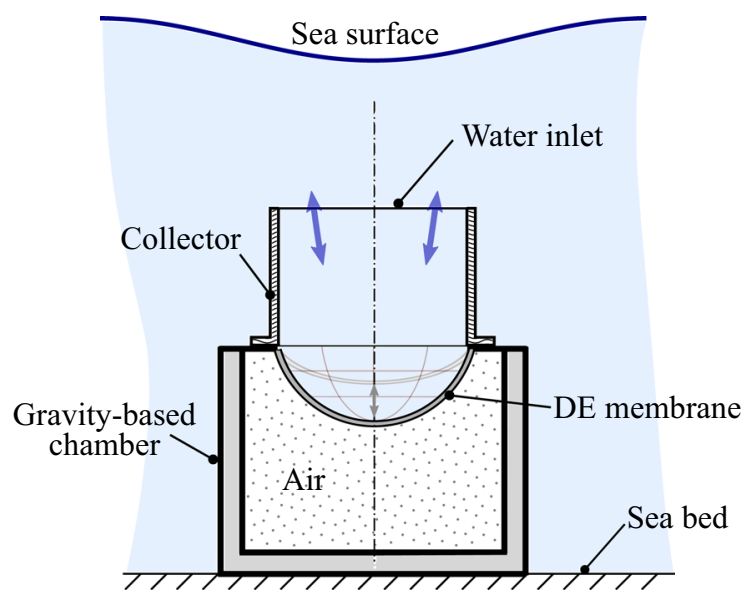

Fig. 1 Architecture of the pressure differential wave energy converter (PD-WEC) equipped with dielectric elastomer power takeoff

merged air chamber. The air chamber is considered here to be rigidly attached to the sea bed, as shown in Fig. 1. Nonetheless, different layouts are possible, e.g. a floating chamber held in position by a mooring system.

The DEG power take-off contacts the air with its bottom face and the sea water with its upper face, and it is surrounded, on the wet side, by a cylindrical collector (or duct) that canalises the sea water towards the DEG. The aim of the collector is to increase the wave excitation force (by increasing the wave pressure at the duct inlet) and the hydrodynamic added mass (i.e. the inertia of the fluid flow within the collector). In the equilibrium configuration, the dielectric elastomer membrane is deformed downward due to the hydrostatic pressure. The mechanical-to-electrical conversion is driven by the wave-induced membrane deformation, which is responsible for the cyclical deformation and variation of the capacitance of the circular diaphragm DEG (CD-DEG). The energy convertible by the DEG through its cyclic deformation increases with the deformation amplitude, the maximum applied electric field and the dielectric permittivity of the elastomeric material $[2,12]$. Splitting the CD-DEG into multiple in-parallel layers (each coated by compliant electrodes) allows the implementation of large electric fields while limiting the applied voltage.

The submerged air chamber can potentially be equipped with an air-pressure regulator. This allows the possibility of exploiting the chamber pressure as a means of adjusting the device stiffness (taking advantage of the nonlinear response of the CD-DEG/air chamber set) and enabling safe operation in the presence of rough sea states.

Even though Fig. 1 represents a single membrane layout, the power take-off can be replaced by an array of smaller membranes per chamber/collector to guarantee ease of installation and replacement in case of failure.

In terms of the dynamic response, the PD-WEC features a negative hydrostatic stiffness, which opposes the positive elastic stiffness of the CD-DEG. The negative hydrostatic stiffness arises since a downward deformation of the membrane corresponds to an increase of the hydrostatic pressure (due to the increased water head above the membrane), which further pushes the downward deformation of the CD-DEG. Thanks to this stiffness compensation mechanism, the PD-WEC can potentially achieve large DEG deformations rather independently of the excitation frequency. Therefore, it offers the potential to efficiently convert wave power in a broad range of incident wave frequencies, with no need for advanced designs aimed at increasing the added mass and achieving resonance. Furthermore, the stiffness compensation principle potentially enables the design of devices capable of large wave-to-wire efficiency within a wide range of dimensions and power targets. This in turn could make it possible to implement PD-WECs with intermediate scale and power output of a few tens of $\mathrm{kW}$ (whose development involves limited risks and costs), in addition to the large power scales typically required for offshore farms.

The CD-DEG employed in the PD-WEC is similar to the power take-off employed in DEG-based oscillating water columns investigated to date [6]. In the latter systems, however, the DEG is installed on top of a collector, out of the water, and its deformation is driven by the pressure of an intermediate air pocket enclosed between the DEG and the free water surface [2]. Since DEG-based oscillating water columns do not benefit from the negative stiffness compensation principle present in PD-WECs, they require optimised hydrodynamic designs (e.g. the inclusion of specifically sized added-mass ducts $[6,7]$ ) to achieve dynamical tuning with the incoming waves, and they only show a resonant response in a rather narrow frequency band.

The development of the PD-WEC brings along technological challenges, primarily related to the adaptation of the DEG power take-off to underwater operation. Making a DEG suitable to operate in direct contact with water is a complex task that requires sophisticated 
engineering. For example, electrical connections and the DEG electrodes need to be safe and watertight.

Some preliminary feasibility studies on the underwater operation of DEGs for wave energy applications have been carried out with reference to other submerged DEG-based WECs [11]. Moreover, several general studies have been carried out to assess the fatigue lifetime of elastomers in water [13] or the influence of the humidity on the electrical properties of dielectric materials [10]. These studies have shown that direct contact with sea water can considerably influence the electromechanical properties and the cyclic lifetime of rubber-like materials. Therefore, dedicated research on materials and technological solutions for the marinisation of DEGs are a prerequisite to the deployment of PD-WEC systems.

\section{Mathematical model}

Modelling a WEC system based on DEG involves three different physical domains to be coupled together: fluid dynamics, solid mechanics and electrostatics. An accurate local description of the problem requires the use of numerical techniques with a large number of degrees of freedom. This approach is computationally onerous in terms of simulation time and computational power. At a preliminary design stage it is preferable to employ lumped-parameter models, which still provide an effective description of the system but allow faster-than-real time simulations.

In this perspective, we hereby present a modelling approach for the PD-WEC in which the system configuration is assumed to be described by one degree of freedom, parametrised through the CD-DEG subtended volume $\Omega_{c}$, shown in Fig. 2. The continuum problem is reduced to one independent variable, and every deformed configuration of the CD-DEG is uniquely identified by one value of $\Omega_{c}$ through a oneto-one mapping procedure. A global energy balance is exploited to derive the PD-WEC equation of motion, following the general approach proposed in [2].

The model relies on the following hypotheses: the hydrodynamics of the system is described by potential flow and linear wave theory; the water velocity inside the collector is considered uniform and parallel to the collector axis, while the water inside the membrane cap is approximated as a solid variable mass; the circular diaphragm DEG is treated as a hyperelastic con-

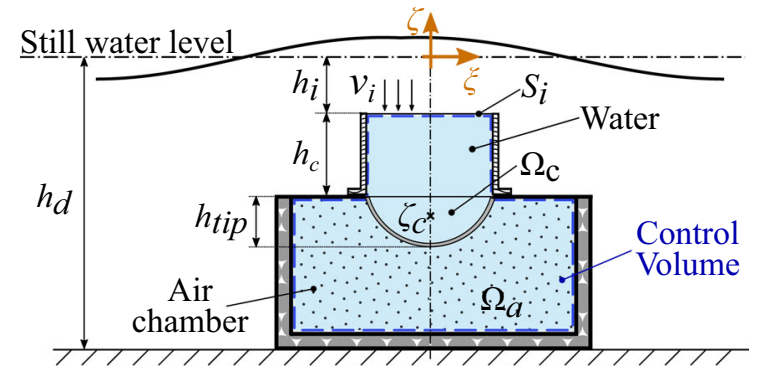

Fig. 2 Dimensions and control volume (blue shaded area) of the PD-WEC. (Color figure online)

tinuum body; the viscosity of the material is neglected; the dielectric elastomer is assumed as a perfect dielectric; the DEG capacitance is equivalent to that of a planar capacitor with non-uniform thickness; and the CDDEG kinetic and gravitational energies are considered negligible compared to those of the moving water volume.

The control volume over which the energy balance is applied is represented by the coloured area in Fig. 2. It includes the air chamber, the CD-DEG and the water volume delimited by the collector side walls and inlet section. The fixed reference frame $\xi-\eta-\zeta$ has the vertical axis lying on the device symmetry axis (positive upward) and the origin lying on the sea still water level.

The following energy balance holds:

$\dot{\mathcal{E}}_{k}+\dot{\mathcal{E}}_{g}+\dot{\mathcal{E}}_{a}+\dot{\mathcal{E}}_{m}+\dot{\mathcal{E}}_{e}=\dot{\mathcal{W}}_{i}+\dot{\mathcal{W}}_{v}+\dot{\mathcal{W}}_{e}$,

where the dot represents differentiation with respect to time and the different terms have the following meaning: $\mathcal{E}_{k}$ is the kinetic energy of the water inside the control volume; $\mathcal{E}_{g}$ is the gravitational potential energy of the water (gravitational potential energy is taken equal to zero in correspondence with the still water level); $\mathcal{E}_{a}$ is the internal energy of the air chamber; $\mathcal{E}_{m}$ is the elastic potential energy of the power take-off; $\mathcal{E}_{e}$ is the electrostatic potential energy of the DEG; $\mathcal{W}_{i}$ is the energy entering the control volume through the inlet section $S_{i} ; \mathcal{W}_{v}$ is the work done on the system by the hydrodynamic viscous forces; and $\mathcal{W}_{e}$ is the electric work supplied to the CD-DEG by the external conditioning electronics.

Due to water incompressibility, the conservation of mass leads to:

$\dot{m}=\rho v_{i} S_{i}=\rho \dot{\Omega}_{c}$,

where $\dot{m}$ is the water mass flow rate through the inlet section (positive if entering), $\rho$ is the water density, 
$v_{i}$ is the water velocity at the inlet section (considered uniform across the area), $S_{i}$ is the cross-sectional area of the duct.

The terms of the energy balance (1) can be specified as follows:

- Kinetic energy. Neglecting the CD-DEG contribution, the kinetic energy of the control volume takes the following form [14]:

$\mathcal{E}_{k}=\frac{1}{2} M_{h} \dot{\Omega}_{c}^{2}$,

where $M_{h}$ (function of $\Omega_{c}$ ) is the generalised hydrostatic inertia of the water volume:

$M_{h}\left(\Omega_{c}\right)=\rho\left(\frac{h_{c}}{S_{i}}+\Omega_{c} \zeta_{c}^{\prime 2}\right)$,

with $\rho$ the water density, $h_{c}$ the duct height, and $\zeta_{c}$ the coordinate of the centre of mass of the water cap contained between the CD-DEG surface and the plane housing its perimeter. Notice that $\zeta_{c}$ is always negative and its value can be uniquely derived from the knowledge of the CD-DEG deformed geometry. The prime symbol' denotes differentiation with respect to $\Omega_{c}$. The first term in Eq. (4) accounts for the inertia of the water within the collector, whereas the second term accounts for the inertia of the water within the DEG cap. Eqs. (3-4) take into account the kinetic energy associated with the movement of the centre of mass of the water cap, but neglect that associated with the movement of the water particles relative to the centre of mass.

- Gravitational potential energy. This is a function of the system geometrical configuration described by $\Omega_{c}$ :

$\mathcal{E}_{g}=\mathcal{E}_{g, 0}+\rho g \Omega_{c} \zeta_{c}$,

where $\mathcal{E}_{g, 0}$ is a constant contribution accounting for the gravitational potential energy of the water inside the collector.

- Air chamber potential energy. The air pocket contained in the control volume is treated as an ideal gas that undergoes an adiabatic transformation. Its internal energy reads as:

$\mathcal{E}_{a}=\frac{p_{0} \Omega_{0}^{\gamma}}{(\gamma-1) \Omega_{a}^{\gamma-1}}$,

where $\Omega_{0}$ and $p_{0}$ are, respectively, the values of the volume and the absolute air pressure of the chamber in the reference equilibrium configuration, $\gamma$ is the adiabatic exponent, and $\Omega_{a}$ is the air chamber volume, namely: $\Omega_{a}=\Omega_{0}+\Omega_{c, 0}-\Omega_{c}$, where $\Omega_{c, 0}$ is the cap volume in the reference equilibrium configuration.

- Elastic energy. The elastic energy of the CD-DEG is expressed as a function of $\Omega_{c}$. Since the CDDEG is usually composed of a stack of identical layers, we define $\mathcal{E}_{m}=N_{l} \mathcal{E}_{m, s}$, where $N_{l}$ is the number of layers and $\mathcal{E}_{m, s}$ is the elastic energy of a single layer. The continuum hyperelastic model that describes the elastic energy $\mathcal{E}_{m}$ is presented in Sect. 3.2.

- Electrostatic energy. The total CD-DEG electrostatic energy is:

$\mathcal{E}_{e}=\frac{1}{2} C V^{2}$,

where $C$ is the total capacitance of the CD-DEG ( $N_{l}$ in-parallel layers) and $V$ is the voltage applied across a single layer. After differentiation with respect to time, Eq. (7) takes the form:

$\dot{\mathcal{E}}_{e}=\frac{1}{2} C^{\prime} V^{2} \dot{\Omega}_{c}+Q \dot{V}$,

where $Q=C V$ is the total charge lying on the in-parallel layers of the stack.

- Electrical power supplied. The electrical power supplied by the external electronics to the CD-DEG is:

$\dot{\mathcal{W}}_{e}=V \dot{Q}=C^{\prime} V^{2} \dot{\Omega}_{c}+Q \dot{V}$

- Viscous energy losses. With reference to the dynamic pressure at the inlet section $S_{i}$, the power loss associated to the hydrodynamic viscous forces can be written as:

$\dot{\mathcal{W}}_{v}=-k_{v} \frac{1}{2} \rho\left|v_{i}\right| v_{i} \dot{\Omega}_{c}=-k_{v} \frac{\rho}{2 S_{i}^{2}}\left|\dot{\Omega}_{c}\right| \dot{\Omega}_{c}^{2}$,

where $k_{v}$ is an unknown viscous coefficient to be calibrated through the experimental results.

- Inlet water flow power. $\dot{\mathcal{W}}_{i}$ takes into account the work done by the water flowing through the inlet section $\left(S_{i}\right)$ of the control volume. This power can be split into three contributions:

$\dot{\mathcal{W}}_{i}=\dot{\Omega}_{c}\left(e_{g}+e_{k}+e_{p}\right)$,

where $e_{g}$ and $e_{k}$ are the gravitational and kinetic energy densities of the flow, i.e.

$e_{g}=-\rho g h_{i}, \quad e_{k}=\frac{1}{2} \rho v_{i}^{2}=\frac{1}{2} \rho\left(\frac{\dot{\Omega}_{c}}{S_{i}}\right)^{2}$,

and $e_{p}$ is the pressure energy at the inlet section:

$e_{p}=p_{\mathrm{atm}}+\rho g h_{i}+p_{w}$, 
where $p_{\text {atm }}$ is the atmospheric pressure, $\rho g h_{i}$ is the hydrostatic pressure and $p_{w}$ is the wave-induced pressure. The last term consists of contributions from the incident, diffracted and radiated waves and is calculated through a wave hydrodynamic model that will be discussed in Sect. 3.1.

Substituting Eqs. $(3,5,8,9,10,11)$ into Eq. (1) leads to the following equation of motion:

$$
\begin{gathered}
M_{h} \ddot{\Omega}_{c}+C_{\Omega} \dot{\Omega}_{c}^{2}+\frac{\rho k_{v}}{2 S_{i}^{2}}\left|\dot{\Omega}_{c}\right| \dot{\Omega}_{c}+\rho g \zeta_{c}^{\prime} \Omega_{c}+\rho g \zeta_{c} \\
+p_{a, r}+\mathcal{E}_{m}^{\prime}-\frac{C^{\prime}}{2} V^{2}=p_{w},
\end{gathered}
$$

where $C_{\Omega}$ is the coefficient of the quadratic term owing to variable inertia and mass flow transportation:

$C_{\Omega}=\frac{1}{2} \rho\left(\zeta_{c}^{\prime 2}+2 \Omega_{c} \zeta_{c}^{\prime} \zeta_{c}^{\prime \prime}-S_{i}^{-2}\right)$,

and $p_{a, r}$ is the relative air chamber pressure:

$p_{a, r}=p_{0}\left(\frac{\Omega_{0}}{\Omega_{0}+\Omega_{c, 0}-\Omega_{c}}\right)^{\gamma}-p_{\text {atm }}$.

Since $\zeta_{c}$ and $\zeta_{c}^{\prime}$ are always negative, the sum of the terms $\rho g \zeta_{c}^{\prime} \Omega_{c}$ and $\rho g \zeta_{c}$ represents a nonlinear negative stiffness contribution. This contribution tends to compensate for the positive stiffness generated by the CD-DEG elasticity (represented by $\mathcal{E}_{m, s}^{\prime}$ ).

The PD-WEC dynamics is described by equation of motion (14) upon specification of the two inputs of the system. The first is the wave excitation pressure $p_{e}$, see Sect. 3.1. The second is represented by an electrical variable of the DEG, whose choice depends on the control strategy adopted. In Eq. (14) and the following, the voltage $V$ is chosen to be user-controlled; therefore, $V$ is treated as an independent variable of the system.

The net electrical power $\dot{\mathcal{W}}_{\text {gen }}$ generated during a deformation is given by the difference between the rate of variation of the energy stored in the CD-DEG and the electrical power supplied to the external electronics, which reads as:

$\dot{\mathcal{W}}_{\text {gen }}=\dot{\mathcal{E}}_{e}-\dot{\mathcal{W}}_{e}=-\frac{1}{2} \dot{C} V^{2}$,

and which shows that electrical energy is positively generated when the CD-DEG capacitance decreases (generator mode), otherwise, electrostatic energy is absorbed by the device (actuator mode).

To solve Eq. (14), a set of complimentary equations are required, and the expression for the wave-induced pressure $p_{w}$ must be derived, as well as $\zeta_{c}, \mathcal{E}_{m}$ and $C$ as functions of $\Omega_{c}$.

\subsection{Hydrodynamic model}

According to the well-established formalism of linear wave theory [9], the wave-induced pressure can be represented by a sum of excitation and radiation contributions:

$p_{w}=p_{e}+p_{r}$,

In the case of point absorbers with small dimensions compared to the wavelength, as in the case of the PD-WEC, the excitation pressure roughly equals the undisturbed wave pressure, with a negligible diffraction contribution. Considering a regular wave, $p_{e}$ is thus obtained by averaging the undisturbed sinusoidal wave pressure $p_{o}$ over the inlet section $S_{i}$ :

$p_{e}=\frac{1}{S_{i}} \iint_{\mathcal{S}} p_{o} \mathrm{~d} \mathcal{S}=\frac{H}{2} \Gamma(\omega) \cos (\omega \tau)$,

where $\tau$ is time, $H$ and $\omega$ are the wave height and angular frequency and $\Gamma(\omega)$ is a frequency-dependent excitation coefficient:

$$
\begin{aligned}
\Gamma(\omega)= & \frac{\rho g}{\pi r_{i}^{2}} \frac{\cosh \left(k_{w}\left(h_{d}-h_{i}\right)\right)}{\cosh \left(k_{w} h_{d}\right)} \times \\
& \times \int_{0}^{2 \pi} \int_{0}^{r_{i}} r \cos \left(k_{w} r \cos \theta\right) \mathrm{d} r \mathrm{~d} \theta,
\end{aligned}
$$

where $k_{w}$ is the wave number, $r_{i}$ is the radius of the collector and the geometric dimensions $h_{i}$ and $h_{d}$ are represented in Fig. 2. This excitation coefficient can be employed to calculate the wave excitation in the presence of panchromatic waves, expressed as a sum of harmonic components with amplitudes distributed according to a spectral model, as described in [7].

Following [15], the radiation contribution is expressed as follows:

$p_{r}=-M_{a d, \infty} \ddot{\Omega}_{c}-\int_{0}^{\tau} k(\tau-t) \dot{\Omega}_{c}(t) \mathrm{d} t$,

where $M_{a d, \infty}$ is the infinite-frequency added mass and the convolution kernel $k(\tau)$ can be expressed in the frequency domain as [16]:

$\mathcal{K}(\omega)=B_{r}(\omega)+i \omega\left(M_{a d}(\omega)-M_{a d, \infty}\right)$,

where $\mathcal{K}(\omega)$ is the Fourier transform of $k(t), i$ is the imaginary unit, $M_{a d}(\omega)$ is the frequency-dependent added mass and $B_{r}(\omega)$ is the radiation damping. Analytical relations are available from literature [16] and can be exploited to determine the coefficients in Eq. (22).

Haskind's equation provides a relation between the excitation coefficient and the radiation damping, which 
for an axisymmetric device takes the following form [9]:

$B_{r}(\omega)=\frac{\omega k_{w} \Gamma^{2}(\omega)}{2 \rho g^{2} \Upsilon}$,

with

$\Upsilon=\left(1+\frac{2 k_{w} h_{d}}{\sinh \left(2 k_{w} h_{d}\right)}\right) \tanh \left(k_{w} h_{d}\right)$.

Exploiting the Kramers-Kronig equations, it is possible to relate the added mass to the radiation damping as follows [9]:

$M_{a d}(\omega)-M_{a d, \infty}=-\frac{2}{\pi} \int_{0}^{+\infty} \frac{B_{r}(v)}{\omega^{2}-v^{2}} \mathrm{~d} v$.

This approach does not provide an explicit equation for the infinite-frequency added mass, which can be determined a posteriori through the experimental results, or via boundary element method codes [17].

A detailed derivation of the excitation pressure as well as a numerical approach to compute the coefficients of the convolution kernel is reported in the Supplementary Material.

\subsection{CD-DEG model}

This section summarises the CD-DEG model necessary to express the elastic energy $\mathcal{E}_{m}$, the capacitance $C$ and the coordinate of the centre of mass of the CD-DEG cap $\zeta_{c}$ as functions of $\Omega_{c}$. These three functions are uniquely identified by the CD-DEG deformed shape, which can be solved for as follows: imagine installing a CD-DEG membrane at the bottom end of a circular tube, and then starting to fill the tube with water from the top (see Fig. 3b). Each value of the water head $h_{0}$ above the plane housing the CD-DEG perimeter is associated with a static deformed shape of the membrane. Analysing the static deformed shapes for different values of $h_{0}$ provides a map for the following quantities: $\mathcal{E}_{m}\left(h_{0}\right), C\left(h_{0}\right), \zeta_{c}\left(h_{0}\right)$ and $\Omega_{c}\left(h_{0}\right)$, where the last function can be employed to express the desired quantities as functions of $\Omega_{c}: \mathcal{E}_{m}\left(\Omega_{c}\right), C\left(\Omega_{c}\right)$ and $\zeta_{c}\left(\Omega_{c}\right)$. This procedure can also be employed to perform laboratory experiments to validate the set of CD-DEG static deformed shapes provided by the model.

The static DEG model proposed herein relies on the following hypotheses:

- The shape of the CD-DEG at a given value of $\Omega_{c}$ is the same regardless of the electrical state and the

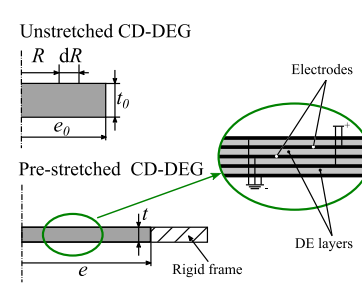

(a)

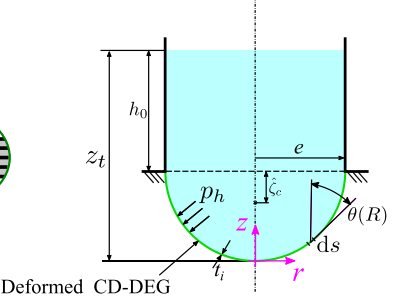

(b)
Fig. 3 a Layout of the circular diaphragm dielectric elastomer generator in its undeformed (top) and pre-stretched (bottom) configurations, respectively. b Static deformed configuration and dimensions

water velocity. This shape is assumed to be the same as that provided by the static CD-DEG response.

- The elastic contribution of the electrodes is neglected and the set of the dielectric elastomer layers are treated as a single thick layer. The incompressible, homogeneous, dielectric material is considered as a lossless hyperelastic continuum: its elastic state is uniquely described by a volumetric strain energy density function $\Psi$ [18].

- The CD-DEG is treated as a purely electrostatic device made by a stack of parallel-plate capacitors (connected in-parallel) with non-uniform thickness.

A static CD-DEG characterisation can be obtained using a FEM software [19] or by direct integration of the equilibrium equations [20]. In this work the second approach is pursued, the details of which are described in the Supplementary Material.

With reference to Fig. 3a, the stack of membranes has radius $e_{0}$ and thickness $t_{0}$ in its undeformed state. The stack is clamped to a rigid frame with a radial prestretch $\lambda_{p}$, which aims to avoid loss of tension induced by electrical activation. The pre-stretched radius and thickness are $e=\lambda_{p} e_{0}$ and $t=\lambda_{p}^{-2} t_{0}$, respectively.

Due to the axial symmetry of the deformation, we employ cylindrical polar coordinates $(R, Z)$ and $(r, z)$ to describe the position of material particles in the undeformed and deformed configuration, respectively (Fig. 3b). In particular, we define a deformed reference frame with the origin located at the CD-DEG tip, which is mobile. This choice simplifies the formulation of boundary conditions and the solution of the equilibrium equations. 
Since in the undeformed configuration $Z=0$ for all points, a material point of the CD-DEG is uniquely identified by the independent variable $R$, which is the distance of the point from the axis in the unstretched state (Fig. 3a). Therefore, the deformation can be expressed as:

$r=r(R), \quad z=z(R)$.

We also introduce a function $\theta(R)$ which represents the angle between the tangent line and the symmetry axis at a particle position $R$ (Fig. 3b). The principal stretches in the meridian, circumferential and thickness direction read as follows:

$\lambda_{1}=\sqrt{r^{\prime 2}+z^{\prime 2}}, \quad \lambda_{2}=\frac{r}{R}, \quad \lambda_{3}=\frac{t_{i}}{t_{0}}=\lambda_{1}^{-1} \lambda_{2}^{-1}$,

where $t_{i}$ is the local CD-DEG thickness in the deformed configuration. In this context the prime symbol denotes differentiation with respect to $R$. The third relation of (27) derives from the material incompressibility.

The pressure difference between the upper and lower face of the CD-DEG, which depends on the coordinate of the membrane, is given by:

$p_{h}=\rho g\left(z_{t}-z\right)$,

where $z_{t}$ (positive) is the distance of the CD-DEG tip from the free water surface (see Fig. 3b).

Following a Lagrangian approach, the system of differential equation governing the mechanical equilibrium of the membrane reads [21]:

$$
\begin{aligned}
\frac{\mathrm{d} \lambda_{1}}{\mathrm{~d} R} & =\frac{\Psi_{2}-\lambda_{1} \Psi_{12}}{R \Psi_{11}} \sin \theta-\frac{\Psi_{1}-\lambda_{2} \Psi_{12}}{R \Psi_{11}}, \\
\frac{\mathrm{d} \lambda_{2}}{\mathrm{~d} R} & =\frac{\lambda_{1} \sin \theta-\lambda_{2}}{R}, \\
\frac{\mathrm{d} \theta}{\mathrm{d} R} & =\frac{\Psi_{2}}{R \Psi_{1}} \cos \theta-\frac{\lambda_{1} \lambda_{2}}{t_{0} \Psi_{1}} p_{h}, \\
\frac{\mathrm{d} z}{\mathrm{~d} R} & =\lambda_{1} \cos \theta,
\end{aligned}
$$

where $\Psi_{i}=\frac{\partial \Psi}{\partial \lambda_{i}}$ and $\Psi_{i j}=\frac{\partial^{2} \Psi}{\partial \lambda_{i} \partial \lambda_{j}}$. A detailed derivation of Eq. (29) is presented in the Supplementary Material.

When the pressure $p_{h}$ is homogenous, i.e. it does not depend on $z,(29)$ is decoupled from the other equations and it can be solved separately. This represents the wellknown case of an air-inflated balloon, for which an approximated model has been presented in the past [7, 22].

To solve the system of equations (29), a modified shooting method is employed. The shooting method technique consists of reducing a boundary value problem to the solution of an initial value problem [23] with four initial conditions. The following initial conditions hold at $R=0$ :

$\lambda_{1}(0)=\lambda_{2}(0)=\lambda_{0}, \quad \theta(0)=\frac{\pi}{2}, \quad z(0)=0$,

where $\lambda_{0}$ is an imposed value of stretch.

The solution steps are as follows: the system of equations (29) is integrated forward from $R=0$ for an imposed value of $\lambda_{0}$ with the unknown parameter $z_{t}$. Then, the boundary condition at $R=e_{0}$ is used to write the constraint equation $\lambda_{2}\left(e_{0}\right)=\lambda_{p}$. Its solution yields the value of $z_{t}$ which verifies the system (29) with the imposed value $\lambda_{0}$. The water head $h_{0}$ is then computed as $h_{0}=z_{t}-z\left(e_{0}\right)$. Repeating the procedure for different values of $\lambda_{0}$ provides the maps of the desired quantities $\mathcal{E}_{m}\left(h_{0}\right), C\left(h_{0}\right), \zeta_{c}\left(h_{0}\right)$ and $\Omega_{c}\left(h_{0}\right)$.

More details about the solution procedure are reported in the Supplementary Material.

Note that the considered model does not involve the solution of a coupled electroelastic problem, since the effect of the electrical activation is tackled separately through the electrical term in the equilibrium equation (14). The solution provided by the CD-DEG model described in this section is an approximation based on the assumption that the CD-DEG deformed shape is represented by a single degree of freedom $\left(\Omega_{c}\right)$. The actual CD-DEG deformed shapes in the presence of the electrical activation are provided by the solution of system (29) with a modified version of the energy function $\Psi$ which accounts for both the deformation and the applied electric field. A comparison between the exact and approximated solutions is presented in the Supplementary Material.

\section{Design of a small-scale prototype}

This section details design criteria of a small-scale PD-WEC prototype for wave tank testing. The wave tank tests considered herein are aimed at the assessment of the fluid-structure response of the PD-WEC and the validation of the dynamic model proposed in Sect. 3. The features validated through the tests are the CD-DEG stiffness compensation principle through the implementation of a negative hydrodynamic stiffness, and the ability of the designed device to achieve large DEG deformations over a wide range of wave frequencies. For the sake of fluid-structure interaction assess- 
ment, tests described herein are run in the absence of electric activation. Feasibility of electric energy generation with small scale DEG-based WEC prototypes has been proved in the past with respect to traditional oscillating water column wave energy converters equipped with a CD-DEG power take-off system [6,7]. Section 4.1 illustrates the scaling rules, in accordance with Froude criteria [24], to consistently project dimensions, dynamic response and performance of a small-scale prototype to a full-scale scenario. Section 4.2 discusses design choices aimed at providing large DEG deformations over a wide range of incident wave frequencies. Section 4.3 describes the manufacturing, testing and data processing procedures.

\subsection{Scaling rules}

Appropriate scaling rules must be applied to project the dimensions, dynamic response and performance of small scale devices (which are employed to conduct laboratory experiments) to real-scale WECs. This is usually achieved by employing the well-established methodology of Froude scaling [24], which states that the ratio of inertial and gravitational forces, namely the Froude number, must remain the same both at small and full scale.

According to Froude rules, each term of the WEC dynamic equilibrium equation (14) must scale with $s_{f}$ in order to perform experiments in conditions of hydrodynamic similarity. The dielectric elastomer material employed is assumed to be the same at both scales and to undergo the same deformations, so that the stretches and the strain energy function are scale invariant. If the geometric dimensions scale by $s_{f}$, all the terms of the energy balance are scaled consistently except for $p_{a, r}$ and $\mathcal{E}_{m}^{\prime}$, which require special attention.

The differentiation of the elastic energy of a single CD-DEG layer is computed as:

$\mathcal{E}_{m}^{\prime}=\frac{\mathrm{d}}{\mathrm{d} \Omega_{c}} \int_{\Omega_{c d}} \Psi\left(\lambda_{1}, \lambda_{2}\right) \mathrm{d} \Omega$,

where $\Omega_{c d}$ is the volume of the dielectric elastomer material. The subtended volume $\Omega_{c}$ scales with $s_{f}^{3}$. Therefore, in order for $\mathcal{E}_{m}^{\prime}$ to scale with $s_{f}$, the dielectric elastomer volume $\Omega_{c d}$ must scale with $s_{f}^{4}$ instead of the geometric factor $s_{f}^{3}$. Since the CD-DEG radius $\left(e_{0}\right)$ has been assumed to scale with $s_{f}$, then the thickness $t_{0}$ must scale with $s_{f}^{2}$ and not with $s_{f}$.
Regarding the air pressure contribution $p_{a, r}$, it turns out that the air chamber volume cannot scale geometrically with $s_{f}^{3}$, as this would prevent the associated air stiffness to scale consistently [24,25]. An approximate relation for a consistent scaling of the air volume (holding for small air variations of the chamber volume) is derived through the linearisation of the adiabatic law $\left(p_{a, r}+p_{\text {atm }}\right) \Omega_{a}^{\gamma}=p_{0} \Omega_{0}^{\gamma}$ (used to model the air behaviour in Eq. (6)):

$p_{a, r} \simeq p_{0, r}-\gamma\left(p_{0, r}+p_{\mathrm{atm}}\right) \frac{\Omega_{c, 0}-\Omega_{c}}{\Omega_{0}}$.

The term $p_{0, r}$ represents the relative air pressure in the reference configuration; since it affects the reference deformed CD-DEG shape, it must scale with $s_{f}$.

The air volume variation $\Omega_{c, 0}-\Omega_{c}$ only depends on the membrane displacement, thus it scales with $s_{f}^{3}$. In conclusion, $\Omega_{0}$ is the only parameter on which it is possible to intervene to make $p_{a, r}$ scale with $s_{f}$. The scaled value of the equilibrium volume $\Omega_{0}^{s}$, which depends on full-scale equilibrium pressure and volume, reads as follows:

$\Omega_{0}^{s}=\Omega_{0} s_{f}^{2}\left(\frac{p_{\mathrm{atm}}+s_{f} p_{0, r}}{p_{\mathrm{atm}}+p_{0, r}}\right)$.

From Eq. (33) it is easy to see that $\Omega_{0}^{s}$ scales with a power factor somewhere between two and three. Since the reference relative pressure $p_{0, r}$ is usually much smaller than the atmospheric pressure, the air volume scaling factor is generally close to $s_{f}^{2}$. According to this result, air volumes required for small-scale experiments are larger than they would be by applying a geometric scaling $s_{f}^{3}$. In the experiments described in the following sections, the small-scale setup has been provided with an additional external air reservoir connected to the air chamber below the CD-DEG by means of a piping system, similar to [24]. In general, connecting two air volumes by means of pipes with smaller diameters with respect to the reservoir dimensions may introduce undesired dynamical effects that are not representative of the dynamics of the full-scale wave energy converter. Therefore, when possible, it is preferable to directly increase the dimension of the principal air tank. In the case of small-scale PD-WEC prototypes; however, using large submerged air chambers would affect the hydrodynamic parameters, leading to an inconsistent scaling. For this reason, in the tests described herein we opted for the employment of an external air expansion vessel. 
Table 1 PD-WEC prototype design features

$\begin{array}{ll}\text { (a) Geometric dimensions } & \\ \text { Inlet sect. depth } & h_{i}=100 \mathrm{~mm} \\ \text { Collector height } & h_{c}=80 \mathrm{~mm} \\ \text { Collector radius } & e=65 \mathrm{~mm} \\ \text { Water depth } & h_{d}=1.15 \mathrm{~m} \\ \text { Air chamber volume* } & 6 \mathrm{dm}^{3} \\ \text { External air tank } & 440 \mathrm{dm}^{3} \\ \text { (b) CD-DEG features } & \\ \text { Material } & \text { TheraBand Rubber } \\ \text { Hyperelastic model } & \text { Gent-Gent } \\ \text { Radius } & e=65 \mathrm{~mm} \\ \text { Thickness } & t_{0}=0.22 \mathrm{~mm} \\ \text { Pre-stretch } & \lambda_{p}=1.6 \\ \text { Number of layers } & N_{l}=1\end{array}$

*Volume of the air chamber with the CD-DEG in the flat configuration

\subsection{Wave tank prototype design}

The wave tank employed to run the test campaign, the Curved Wave Tank facility at Edinburgh University, has an operating wave frequency range approximately lying between 0.6 and $1.2 \mathrm{~Hz}$. This constraint sets the approximate scaling factor for the tested prototype with respect to a hypothetical full-scale installation. Indicatively, considering that common sea states have wave frequencies generally comprised in the range $0.07-0.25 \mathrm{~Hz}$, prototypes tested in this facility can be assumed to feature a scaling factor between 1/30 and 1/40, based on Froude scaling criteria [7].

As a first design step, the principal geometric features have been identified such that the PD-WEC static equilibrium configuration lies in a low stiffness region of the static response, so that small pressure variations produce large CD-DEG deformations. At this stage, considerations on the commercial materials for the construction of the small-scale prototype have also been taken into account. Following that, the PD-WEC dynamic behaviour has been considered, with the aim of ensuring that the device provides large DEG deformations within the frequency range of the tank facility. A sensitivity analysis of the PD-WEC dynamic response with respect to the wave excitation amplitude and frequency is performed. Different simulations are run to choose the design values of the collector height $h_{c}$, the inlet section depth $h_{i}$ and the CD-DEG prestretch $\lambda_{p}$. The chosen design parameters are listed in Table 1.

The chosen dielectric elastomer material is a custom batch styrene-based rubber produced by TheraBand ${ }^{\circledR}$. It exhibits good performance as a result of a flat trend in its stress-strain curve [10] (i.e. a low-stiffness) over a wide range of stretch values (between 1.5 and 3), which results in large capacitance variations. The physical properties of a similar material have been investigated in [10]. Compared to the rubber investigated in [10], however, the custom rubber employed here features larger dimensions of the scratch membrane roll (so as to match the dimensions of the PD-WEC prototype) and is manufactured without pigment inclusion (which leads to different elastic properties). Its elastic behaviour is well fitted by a Gent-Gent hyperelastic strain energy function [26]:

$$
\begin{aligned}
\Psi\left(\lambda_{1}, \lambda_{2}\right)=- & \frac{\mu}{2} J_{m} \log \left(1-\frac{\lambda_{1}^{2}+\lambda_{2}^{2}+\lambda_{1}^{-2} \lambda_{2}^{-2}-3}{J_{m}}\right) \\
& +C_{2} \log \left(\frac{\lambda_{1}^{-2}+\lambda_{2}^{-2}+\lambda_{1}^{2} \lambda_{2}^{2}}{3}\right),
\end{aligned}
$$

where the constitutive parameters have been derived from static tensile tests, and they read as follows: $\mu=$ $132 \mathrm{kPa}, J_{m}=45$ and $C_{2}=10 \mathrm{kPa}$.

Mechanical and electrical properties of dielectric materials and electrodes may be affected by temperature changes [27], which can take place in the sea environment. However, the aim of this work is the validation of the PD-WEC design, mainly centred on the negative stiffness compensation mechanisms. The electrical and mechanical material properties have been thus assumed constant with temperature.

With a scale factor between $1 / 30$ and $1 / 40$, the smallscale prototype would translate into a real-scale device whose CD-DEG diameter is somewhere between 6 and 8 meters, with a mass of dielectric elastomer material in the order of 6-7 tons. Considering conservative levels of dielectric elastomer energy density already achieved in recent studies [6,7], a power output of a few hundreds of kilowatts can be expected for the real-scale wave energy converter.

\subsection{Manufacturing and testing}

The setup consists of four main parts: the PD-WEC device, a support structure, a gravity foundation and 
an external air reservoir for air chamber stiffness scaling, as shown in Fig. 4a. The cylindrical-shaped wall of the air chamber has been constructed from acrylic plastic (polymethyl methacrylate) with external diameter of $200 \mathrm{~mm}$ and thickness of $3 \mathrm{~mm}$. The top and bottom plates used to close the air chamber and the flange employed to anchor the membrane have been built in aluminium alloy. The upper collector has been manufactured as a single 3D-printed piece (ABS plastic). The CD-DEG pre-stretched membrane is flanged between the two rings, and all the connections are properly sealed to avoid air leaks. The device is held in the design position by means of a beam structure, which is equipped with a ballast mass at its bottom to prevent movements due to the wave action. A commercial plastic hose is used to connect the air chamber to the external air reservoir.

The wave tank employed for the test campaign features a semicircular surface with diameter of $9 \mathrm{~m}$ and water depth of $1.2 \mathrm{~m}$. Waves are generated by 48 wave makers distributed along the semi-circumference, see Fig. 4b. Experiments were conducted in a wide set of regular waves with frequency ranging between 0.5 and $1.2 \mathrm{~Hz}$ (corresponding to wave periods of $4.5-12.5 \mathrm{~s}$ at a scale 30/40 times larger) and height $H$ in the range $80-120 \mathrm{~mm}$ (i.e. $1.2-4.8 \mathrm{~m}$ full scale equivalent). The resulting experimental data have been used to validate the proposed model. In addition to that, a restricted set of tests have been carried out in irregular wave conditions (with a JONSWAP spectral distribution [28]). Moreover, a series of tests without the upper collector $\left(\mathrm{h}_{c}=0\right)$ have been run in order to assess the influence of the collector on the PD-WEC dynamic response.

For each regular wave test, data acquisitions of about $20 \mathrm{~s}$ have been performed after steady-state conditions were reached. For irregular tests, the duration of the acquisitions was about 100 seconds.

The air chamber pressure and the membrane deformed shape have been measured during the experiments. The value of the pressure inside the submerged air chamber has been acquired through a MPX12 Freescale Semiconductor ${ }^{\circledR}$ sensor at a sampling frequency of $1 \mathrm{kHz}$. The deformation of the membrane has been captured through video recording. A Point Grey camera model GS3-U3-23S6M-C with lens LENS250F6C has been employed, together with the proprietary acquisition software FlyCapture 2.9. Video frames were recorded at a rate of $100 \mathrm{fps}$. The camera was placed near the glass wall of the tank, approx-

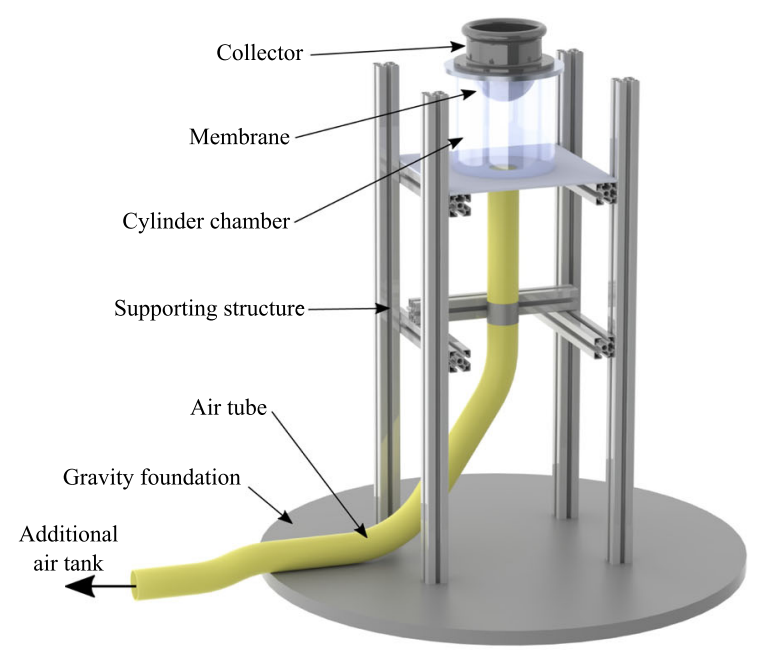

(a)

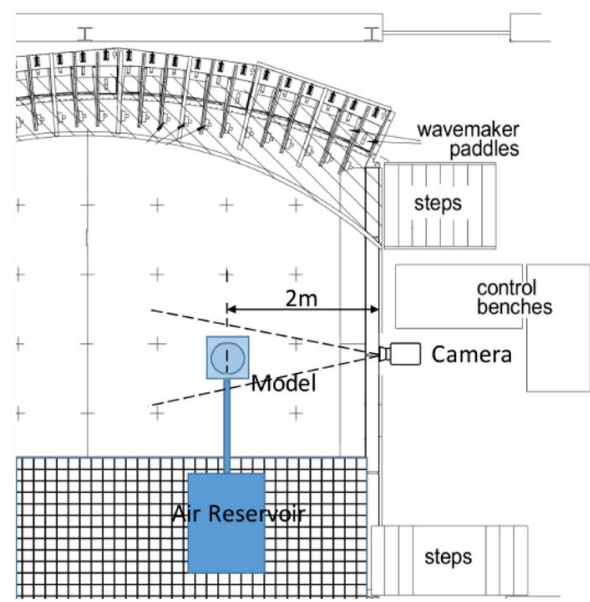

(b)

Fig. 4 a Render of the PD-WEC prototype used during the experimental tests (the circular diaphragm membrane has been represented in its inflated configuration for illustrative purposes, though it holds a flat position at atmospheric pressure). b Plan view schematic representation of part of the wave tank facility with the installed PD-WEC prototype

imately at the same height of, and pointing toward, the PD-WEC (see Fig. 4b). Three spherical optical markers (visible in Fig. 5) have been employed to accurately estimate the relative position of the PD-WEC with respect to the camera position. Figure 5 shows a frame series of a video recording. During the postprocessing data analysis, the membrane tip elevation has been identified using a sequence of MATLAB ${ }^{\circledR}$ image filters, as previously described in [6]. 

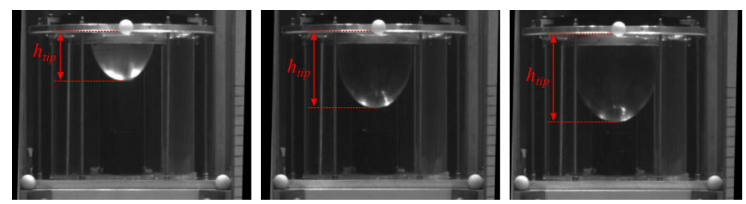

Fig. 5 Three different frames of the high-speed camera video acquisition. The optical markers are visible in the bottom left, bottom right and top centre of the frames

\section{Results}

This section presents the results of the experimental tests. Section 5.1 shows some relevant time series measured in different operating conditions; Sect. 5.2 compares the experimental data with the model predictions, demonstrating that the model is able to efficiently describe the nonlinear PD-WEC dynamics.

\subsection{Overview of results}

In the following we present some relevant time series of the air chamber pressure and membrane tip elevation for different sea states and different arrangements of the device, i.e. with and without the upper collector.

As an example, Fig. 6 shows the response of the system (namely the air chamber relative pressure and the CD-DEG tip height below its perimeter) in two different conditions: a regular sea state with wave frequency equal to $0.8 \mathrm{~Hz}$ and wave height of $120 \mathrm{~mm}$; and an irregular sea state, with wave peak frequency of $0.8 \mathrm{~Hz}$ and significant wave height of $80 \mathrm{~mm}$. Both pressure and tip elevation time series in Fig. 6 have been offset in a way that each curve has time average equal to zero.

When the upper collector is absent (blue solid lines), the oscillation amplitude of both signals is smaller (both in regular and irregular waves) as a result of the lower excitation load. In the absence of the collector, the system has lower hydrodynamic inertia (i.e. $h_{c}=0$ in Eq. (4)) and, hence, higher natural frequency. As a consequence, in the absence of the collector, a significant rebound is present in the membrane tip displacement (blue line in Fig. 6c). This corresponds to an undamped oscillation of the system at its natural frequency that, due to the low inertia, nearly doubles the excitation frequency.

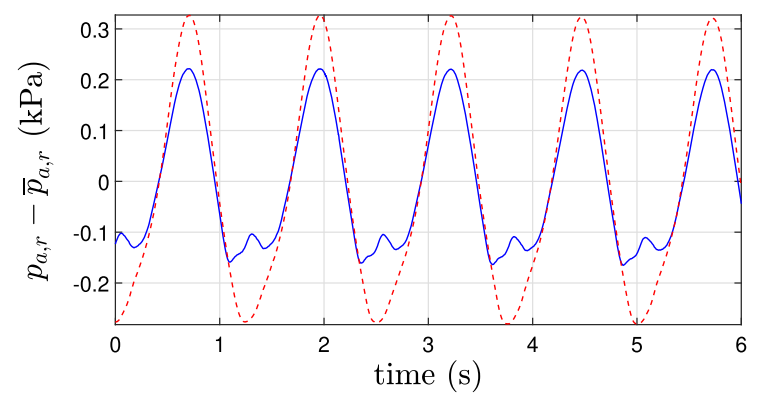

(a)

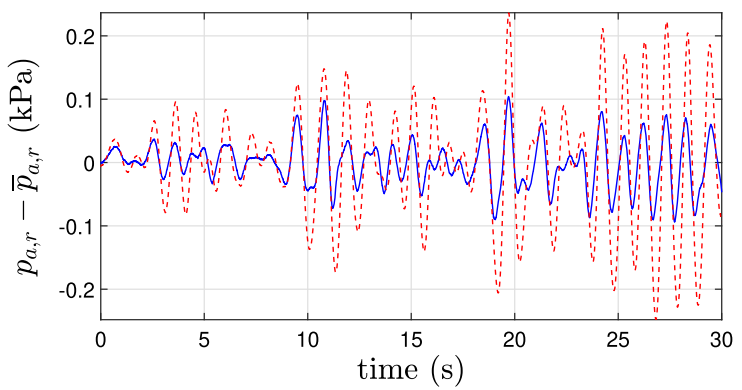

(b)

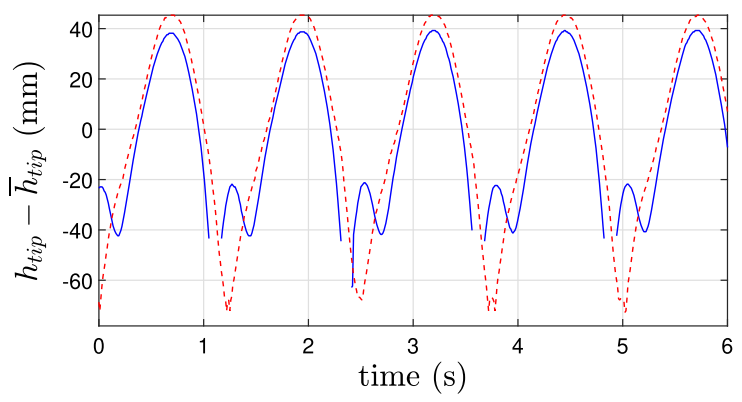

(c)

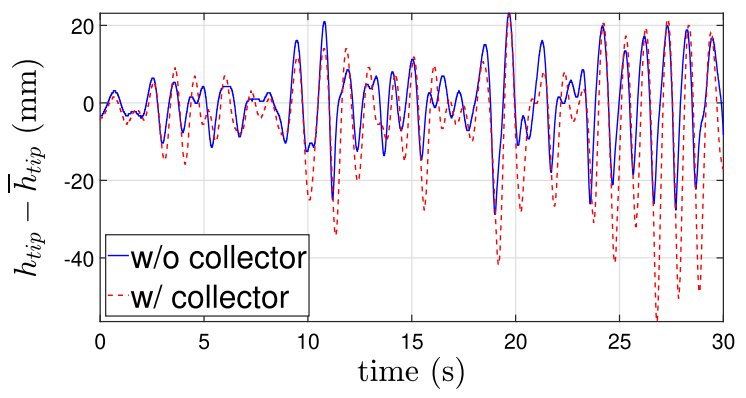

(d)

Fig. 6 Times-series acquisitions with and without upper collector (dashed and solid curves, respectively). a, b The de-averaged air chamber pressure oscillation for regular and irregular wave tests, respectively. c, d The corresponding de-averaged membrane tip oscillation. The notation $\bar{x}$ represents the time-averaged value of the the generic signal $x(t)$ over the time interval shown in the figure. (Color figure online) 


\subsection{Model validation}

This section presents the validation of the nonlinear PD-WEC model against experimental results for regular wave conditions in the presence of the upper collector. Numerical solutions are obtained by implementing the presented set of equations in a MATLAB and Simulink ${ }^{\circledR}$ environment.

The air chamber pressurisation as well as the device draft (i.e. the reference equilibrium configuration) has been kept constant throughout the different runs. The relative air chamber pressure in the reference configuration $p_{0, r}$ has been set to $0.9 \mathrm{kPa}$.

The viscous coefficient $k_{v}$ and the infinite-frequency added mass $M_{a d, \infty}$, which are not known in advance, have been calibrated by exploiting the experimental results.

For every frequency tested at a wave height equal to $120 \mathrm{~mm}$, several numerical simulations have been run for different values of $k_{v}$ and $M_{a d, \infty}$. Then, the optimal values of the parameters have been chosen so as to minimise the total average difference between model and experimental steady-state oscillation amplitudes. A value of $\mathrm{k}_{v}=3$ has been identified, while $M_{a d, \infty}$ has been set equal to the mass of the control volume in the reference equilibrium configuration, $M_{h}\left(\Omega_{c, 0}\right)$, which means that the total water displaced at the highest frequencies is twice that of the control volume in the reference configuration.

Upon model calibration, the model outcomes are compared with experimental results for a large set of monochromatic sea states. A comparison of the experimental and the model-predicted time series of the air chamber pressure and CD-DEG tip displacement for a wave height of $120 \mathrm{~mm}$ and different wave frequencies is presented in Fig. 7a, b, respectively. The time series show discernible asymmetries in the upwards and downwards deformations (due to nonlinearity), which the model is able to capture.

Figure 8 shows the maxima and minima of pressure and tip height oscillations for different wave amplitudes and frequencies, comparing the experimental results and the model predictions. Upward and downward oscillation extremes are shown separately, since they differ due to the system nonlinearities (nonlinear inertia, quadratic forces, membrane stiffness, etc.). The plots show that the maxima and minima of the CD-DEG deformation are nearly independent of the excitation frequency, and that the CD-DEG reaches large defor-

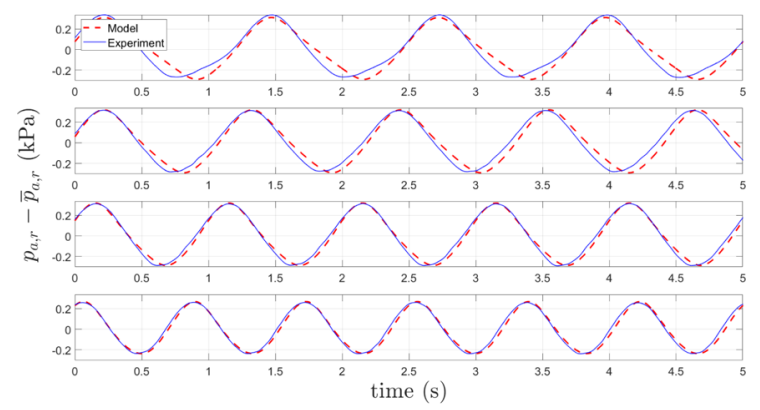

(a)

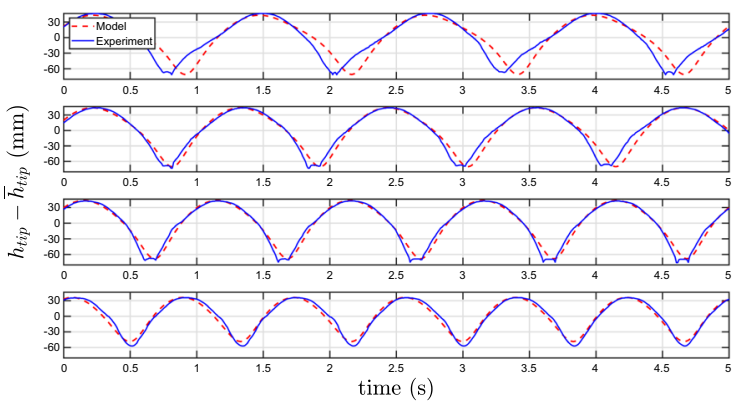

(b)

Fig. 7 Comparison between experimental time series (blue lines) and model time series (dashed red lines) of a the relative pressure inside the air chamber, and $\mathbf{b}$ the membrane tip displacement. Oscillations with respect to the time-averaged value are reported. Tests are run with a wave height of $120 \mathrm{~mm}$ and frequencies (from top to bottom graphs), respectively, equal to $0.8,0.9,1,1.2 \mathrm{~Hz}$. (Color figure online)

mations over the entire frequency range (e.g. with wave heights of $120 \mathrm{~mm}$, the crest-to-trough tip displacement is nearly equal to the CD-DEG pre-stretched diameter). In a functioning system, this is expected to lead to efficient wave power capture, upon control and electrical activation of the DEG power take-off.

In addition to that, Fig. 8 demonstrates a good agreement between experimental data and predicted values, with the model generally predicting slightly smaller deformations with respect to the experiments.

In order to quantitatively evaluate the performance of the model, an average error has been defined as follows: at a given wave height and for each frequency, the relative difference between experimental and modelpredicted oscillation amplitudes has been computed for both pressure and tip displacement signals. The errors have then been averaged over the frequency, obtaining a global error that is only a function of the wave height. For a wave height of $120 \mathrm{~mm}$ the error is about $3.7 \%$ for pressure and $8.3 \%$ for tip displacement, while for 
wave height of $80 \mathrm{~mm}$ the errors rise to $11.6 \%$ and $19 \%$ for pressure and tip displacement, respectively.

Both pressure and tip displacement errors tend to increase when the wave height decreases. A possible reason for this is the approximate hydrodynamic loss model, which makes use of a constant viscous coefficient $k_{v}$, the value of which has been calibrated using the dataset with the largest wave height.

In terms of general trend, the static membrane model provides a good estimate of both the offset equilibrium position and the DEG elastic stiffness. Despite the errors and the large number of underlying assumptions, the model efficiently describes the dynamics of the system. Although a more accurate calculation of the involved physical parameters might be achieved with advanced computationally expensive numerical methods, the proposed model can serve as an effective tool to gain prompt insight on the orders of magnitude of concurrent phenomena and parameters, e.g. membrane stiffness, hydrostatic and air rigidity, influence of the geometric features, etc.

It is worth remarking that the membrane static position and its load-deformation characteristic are strongly sensitive to the constitutive hyperelastic parameters. Therefore, in order to reliably describe and simulate the hydroelastic response of the PD-WEC system, an accurate mechanical characterisation of the dielectric elastomer material properties is key.

One limit of the presented modelling approach comes from the assumption that the membrane kinematics is described by a single degree of freedom. Future improvements may include multi-degree of freedom models. In that case, the CD-DEG deformation can be described through a generalised coordinate approach. The multi-degree of freedom generalised coordinate approach can also be employed to compute the hydrodynamic coefficients exploiting a boundary element method, as detailed in the Supplementary Material.

The viscosity of the dielectric elastomer material has not been taken into account, which is a reasonable assumption when low-hysteresis materials are employed. In practice, it is expected that future dielectric elastomer materials, of practical interest in largescale energy harvesting systems, will feature low viscosity, adequate electromechanical lifetime, enhanced dielectric properties, and a high-quality defect-free manufacturing [10].

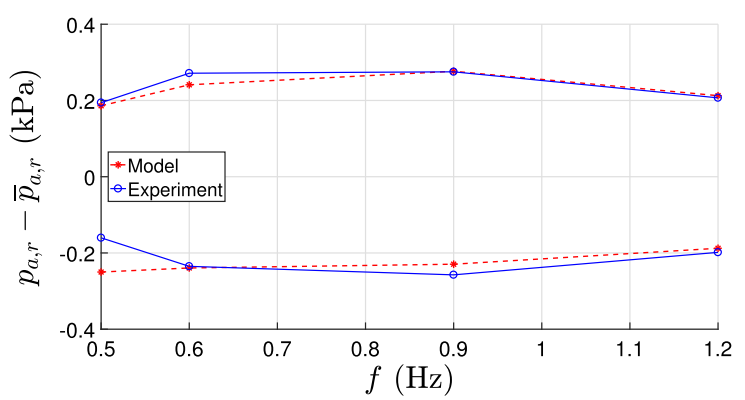

(a) $H=80 \mathrm{~mm}$

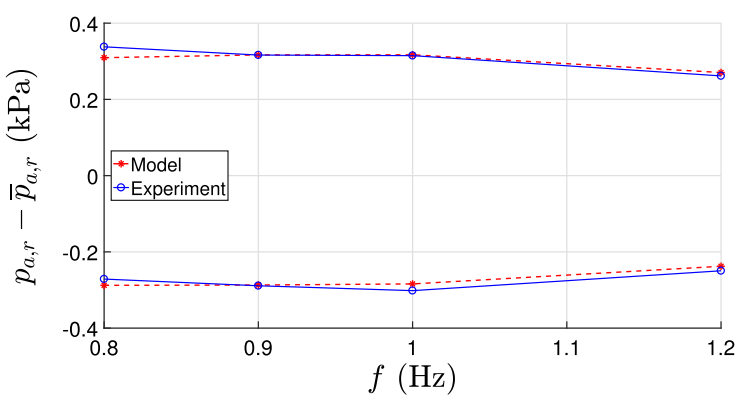

(b) $H=120 \mathrm{~mm}$

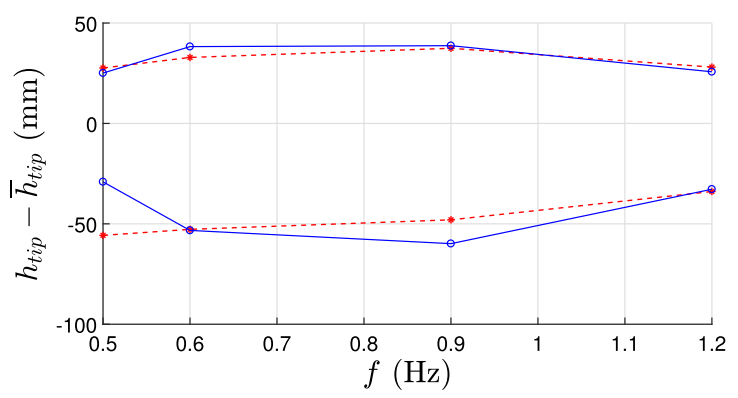

(c) $H=80 \mathrm{~mm}$

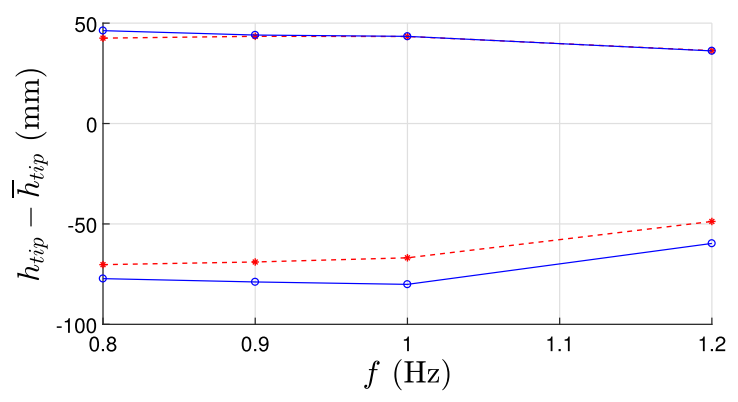

(d) $H=120 \mathrm{~mm}$

Fig. 8 Comparison of the experimental and model predicted maxima and minima in the oscillations of the air pressure and membrane tip height (with respect to the time-averaged value) at different wave frequencies, $f$. a, c Refer to tests with wave height of $80 \mathrm{~mm}$, while $\mathbf{b}, \mathbf{d}$ refer to tests with wave height of $120 \mathrm{~mm}$ 


\section{Conclusions}

This paper presents a new concept of wave energy converter (WEC) featuring a power take-off system based on dielectric elastomer generators (DEGs). DEGs are electrostatic compliant transducers that can convert mechanical energy into direct-current electrical energy exploiting the capacitance variation of a deformable elastomeric capacitor.

The analysed WEC concept, referred to as a pressure differential WEC (PD-WEC), differs from previously proposed DEG-based solutions, as it features a DEG power take-off whose deformation is directly driven by the hydrostatic and hydrodynamic pressures due to direct contact between the DEG and the water waves. In particular, the PD-WEC consists of a submerged air chamber, closed on the upper surface by a DEG membrane, on top of which a water column is present. Wave-induced pressures induce cyclic DEG deformations and consequent capacitance variations which allow the achievement of direct electric power generation.

In terms of the system dynamical response, the PD-WEC layout implements a negative hydrodynamic stiffness (associated with the displacements of the water column above the DEG), which combines with the additional positive stiffness (introduced by the presence of the DEG) to produce a more favourable total stiffness. This stiffness compensation principle makes it possible to design devices with limited dimensions capable of providing large DEG deformations (and power capture) over a wide range of sea conditions, while rejecting strict requirements for dynamical tuning and resonant designs typical of other WEC architectures.

A mathematical model of the PD-WEC is presented, which relies on a nonlinear time-domain formulation derived from a global energy balance. The hydrodynamic model is based on linear wave theory, whereas the DEG electroelastic response is modelled through a reduced one degree of freedom model, in which the DEG material is treated as a homogeneous hyperelastic dielectric. Despite the numerous simplifications, the model takes into account several nonlinearities and enables computationally inexpensive simulation of the fluid-structure interaction between the DEG and the fluid domain.

A small-scale prototype has been designed and tested in a wave tank facility in regular and irreg- ular waves, with the aim of characterising the PDWEC hydroelastic response. The experiments proved the ability of the designed prototype to consistently reach large DEG deformations over a wide range of wave frequencies. Moreover, experimental results also allowed the calibration and validation of the proposed mathematical model, proving its ability to capture the main features of the PD-WEC nonlinear dynamics.

To further investigate the potential of the PD-WEC, power generation tests with fully functional DEG prototypes will be performed in the future. To mitigate the risks due to the application of high-voltage to a submerged DEG, dedicated test-benches will be developed as an alternative to wave tank tests. These test benches should demonstrate the ability to replicate the dynamic loads and operating conditions measured through the wave tank tests described, while suppressing the risks and the uncertainties typical of these wave tank experiments.

Acknowledgements The authors are grateful to TheraBand ${ }^{\circledR}$ (Akron, $\mathrm{OH}$ ) who kindly produced and provided custom elastomeric materials for the tests. Furthermore, the authors would like to thank Mr. Jean-Baptiste Richon (The University of Edinburgh) and Mr. Francesco Damiani (Scuola Superiore Sant'Anna) for their invaluable help during the experimental tests and Dr. Gastone Pietro Rosati Papini (University of Trento) who helped in processing the high-speed camera frames.

Funding Open access funding provided by Scuola Superiore Sant'Anna within the CRUI-CARE Agreement. The research presented in this paper received funds from the Wave Energy Scotland, PTO programme (project "Direct contact Dielectric Elastomer").

\section{Declarations}

Conflict of interest The authors declare that they have no conflict of interest.

Open Access This article is licensed under a Creative Commons Attribution 4.0 International License, which permits use, sharing, adaptation, distribution and reproduction in any medium or format, as long as you give appropriate credit to the original author(s) and the source, provide a link to the Creative Commons licence, and indicate if changes were made. The images or other third party material in this article are included in the article's Creative Commons licence, unless indicated otherwise in a credit line to the material. If material is not included in the article's Creative Commons licence and your intended use is not permitted by statutory regulation or exceeds the permitted use, you will need to obtain permission directly from the copyright holder. To view a copy of this licence, visit http://creativecommons.org/licenses/ by $/ 4.0 /$. 


\section{References}

1. Carpi, F., De Rossi, D., Kornbluh, R., Pelrine, R.E., SommerLarsen, P.: Dielectric Elastomers as Electromechanical Transducers: Fundamentals, Materials, Devices, Models and Applications of an Emerging Electroactive Polymer Technology. Elsevier, New York (2011)

2. Moretti, G., Herran, M.S., Forehand, D., Alves, M., Jeffrey, H., Vertechy, R., Fontana, M.: Advances in the development of dielectric elastomer generators for wave energy conversion. Renew. Sustain. Energy Rev. 117, 109430 (2020)

3. Chiba, S., Waki, M., Wada, T., Hirakawa, Y., Masuda, K., Ikoma, T.: Consistent ocean wave energy harvesting using electroactive polymer (dielectric elastomer) artifficial muscle generators. Appl. Energy 104, 497-502 (2013)

4. Kaltseis, R., Keplinger, C., Koh, S.J.A., Baumgartner, R., Goh, Y.F., Ng, W.H., Kogler, A., Tröls, A., Foo, C.C., Suo, Z., et al.: Natural rubber for sustainable high-power electrical energy generation. RSC Adv. 4(53), 27905-27913 (2014)

5. Ahnert, K., Abel, M., Kollosche, M., Jørgensen, P., Kofod, G.: Soft capacitors for wave energy harvesting. J. Mater. Chem. 21(38), 14492-14497 (2011)

6. Moretti, G., Papini, G.P.R., Righi, M., Forehand, D., Ingram, D., Vertechy, R., Fontana, M.: Resonant wave energy harvester based on dielectric elastomer generator. Smart Mater. Struct. 27(3), 035015 (2018)

7. Moretti, G., Rosati Papini, G.P., Daniele, L., Forehand, D., Ingram, D., Vertechy, R., Fontana, M.: Modelling and testing of a wave energy converter based on dielectric elastomer generators. Proc. R. Soc. A 475(2222), 20180566 (2019)

8. Moretti, G., Malara, G., Scialò, A., Daniele, L., Romolo, A., Vertechy, R., Fontana, M., Arena, F.: Modelling and field testing of a breakwaterintegrated U-OWC wave energy converter with dielectric elastomer generator. Renew. Energy 146, 628-642 (2020)

9. Falnes, J.: Ocean Waves and Oscillating Systems: Linear Interactions Including Wave Energy Extraction. Cambridge University Press, Cambridge (2002)

10. Chen, Y., Agostini, L., Moretti, G., Fontana, M., Vertechy, R.: Dielectric elastomer materials for large-strain actuation and energy harvesting: a comparison between styrenic rubber, natural rubber and acrylic elastomer. Smart Mater. Struct. 28(11), 114001 (2019)

11. Jean, P., Wattez, A., Ardoise, G., Melis, C., Van Kessel, R., Fourmon, A., Barrabino, E., Heemskerk, J., Queau, J.P.: Standing wave tube electro active polymer wave energy converter. In: Electroactive Polymer Actuators and Devices (EAPAD) 2012. vol. 8340, p. 83400C. International Society for Optics and Photonics (2012)

12. Koh, S.J.A., Keplinger, C., Li, T., Bauer, S., Suo, Z.: Dielectric elastomer generators: how much energy can be converted? IEEE/ASME Trans. Mechatron. 16(1), 33-41 (2011)

13. Le Gac, P.-Y., Arhant, M., Davies, P., Muhr, A.: Fatigue behavior of natural rubber in marine environment: comparison between air and sea water. Mater. Des. (1980-2015) 65, 462-467 (2015)

14. Goldstein, H., Poole, C., Safko, J.: Classical mechanics. In: AAPT (2002)
15. Babarit, A., Hals, J., Muliawan, M.J., Kurniawan, A., Moan, T., Krokstad, J.: Numerical benchmarking study of a selection of wave energy converters. Renew. Energy 41, 44-63 (2012)

16. Alves, M.: Numerical simulation of the dynamics of point absorber wave energy converters using frequency and time domain approaches. Universidade Tecnica de Lisboainstituto Superior Tecnico (2012)

17. Newman, J.N., Lee, C.-H.: Boundary-element methods in offshore structure analysis. J. Offshore Mech. Arctic Eng. 124(2), 81-89 (2002)

18. Holzapfel, G.A.: Nonlinear solid mechanics: a continuum approach for engineering science. Meccanica 37(4), 489490 (2002)

19. Vertechy, R., Frisoli, A., Bergamasco, M., Carpi, F., Frediani, G., De Rossi, D.: Modeling and experimental validation of buckling dielectric elastomer actuators. Smart Mater. Struct. 21(9), 094005 (2012)

20. Yang, W.H., Feng, W.W.: On axisymmetrical deformations of nonlinear membranes. J. Appl. Mech. 37(4), 1002-1011 (1970)

21. Zhou, L., Wang, S., Li, L., Fu, Y.: An evaluation of the Gent and Gent-Gent material models using in ation of a plane membrane. Int. J. Mech. Sci. 146, 39-48 (2018)

22. Righi, M., Vertechy, R., Fontana, M.: Experimental characterization of a circular diaphragm dielectric elastomer generator. In: Proceedings of the ASME 2014 Conference on Smart Materials, Adaptive Structures and Intelligent Systems, pp. 8-10. American Society of Mechanical Engineers, Newport, RI, USA (2014). https://doi.org/10.1115/ SMASIS2014-7481

23. Vetterling, W.T., Teukolsky, S.A., Press, W.H., Flannery, B.P.: Numerical Recipes: The Art of Scientifc Computing, 12th edn. Cambridge University Press, Cambridge (1992)

24. Falcão, A.F.O., Henriques, J.C.C.: Model-prototype similarity of oscillating-watercolumn wave energy converters. International Journal of Marine Energy 6, 18-34 (2014)

25. Falcao, A.F.O., Henriques, J.C.C.: The spring-like air compressibility effect in oscillating-water-column wave energy converters: review and analyses. Renew. Sustain. Energy Rev. 112, 483-498 (2019)

26. Steinmann, P., Hossain, M., Possart, G.: Hyperelastic models for rubberlike materials: consistent tangent operators and suitability for Treloar's data. Arch. Appl. Mech. 82(9), 1183-1217 (2012)

27. Khajehsaeid, H., Azar, H.B.: Influence of stretch and temperature on the energy density of dielectric elastomer generators. Appl. Math. Mech. 40(11), 1547-1560 (2019)

28. Pecher, A., Kofoed, J.P.: Handbook of Ocean Wave Energy. Springer, London (2017)

Publisher's Note Springer Nature remains neutral with regard to jurisdictional claims in published maps and institutional affiliations. 